\title{
The Validity and Reliability of Characterizing Epilepsy Based on an External Review of Medical Records
}

\author{
Bong Su Kang ${ }^{1}$, Hae-Kwan Cheong ${ }^{2}$, Ki-Young Jung ${ }^{3}$, Sang Hyeon Jang ${ }^{4}$, Jae Kook Yoo ${ }^{3}$, \\ Dong Wook Kim ${ }^{5}$, Soo-Eun Chung ${ }^{2}$, Seo-Young Lee ${ }^{6}$ \\ 1Department of Neurology, Seoul National University Hospital, Seoul; '2Department of Social and Preventive Medicine, Sungkyunkwan University

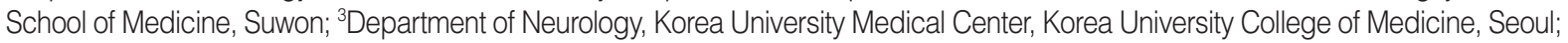 \\ ${ }^{4}$ Department of Neurology, Eulji University College of Medicine, Daejeon; ${ }^{5}$ Department of Neurology, Konkuk University School of Medicine, \\ Seoul; ${ }^{6}$ Department of Neurology, Kangwon National University School of Medicine, Chuncheon, Korea
}

OBJECTIVES: Our goal is to validate diagnosing and characterizing epilepsy based on a medical record survey by external reviewers.

METHODS: We reviewed medical records from 80 patients who received antiepileptic drugs in 2009 at two hospitals. The study consisted of two steps; data abstraction by certified health record administrators and then verification by the investigators. The gold standard was the results of the survey performed by the epileptologists from their own hospital.

RESULTS: The specificity was more than $90.0 \%$ for diagnosis and activity, and for new-onset seizures. The sensitivity was $97.0 \%$ or more for diagnosis and activity and $66.7-75.0 \%$ for new-onset epilepsy. This method accurately classified epileptic syndromes in $90.2-92.9 \%$ of patients, causes in $85.4-92.7 \%$, and age of onset in $78.0-81.0 \%$. Kappa statistics for inter-rater reliability and test-retest reliability ranged from $0.641-0.975$, which means substantial to near-perfect agreement in all items.

CONCLUSIONS: Our data suggest that epilepsy can be well identified by external review of medical records. This method may be useful as a basis for large-scale epidemiological research.

KEY WORDS: Validity, Epilepsy, Epidemiology, Sensitivity, Specificity, Reliability

\section{INTRODUCTION}

The Korean National Health Insurance (NHI) has provided health care for the entire Korean population as well as all medical facilities since 1989 and its database is a useful source of data for epidemiological research. Our previous study demon-

\section{Correspondence: Seo-Young Lee, MD, PhD}

Department of Neurology, Kangwon National University School of Medicine, 156 Baengnyeong-ro, Chuncheon 200-722, Korea

Tel: +82-33-258-2431, Fax: +82-33-258-2103

E-mail: leeseoyoung@kangwon.ac.kr

Received: Feb 6, 2013, Accepted: Jul 4, 2013, Published: Aug 23, 2013

This article is available from: http://e-epih.org/.

(C) 2013, Korean Society of Epidemiology

(C) This is an open-access article distributed under the terms of the Creative Commons Attribution License (http://creativecommons.org/licenses/by/3.0/), which permits unrestricted use, distribution, and reproduction in any medium, provided the original work is properly cited. strated its value in estimating the national prevalence of epilepsy based on antiepileptic drugs (AEDs) prescribed and diagnostic codes for claims [1]. However, NHI data should be validated for epidemiological research [2,3]. In addition, the NHI data do not provide detailed clinical information. Therefore, we launched the Epidemiological Study of Seizure and Epilepsy using Nationwide database for Corean Epilepsy patients (ESSENCE) project to estimate the prevalence of epilepsy from NHI data, which were validated and supplemented by a review of medical records.

Surveys reviewing medical records hold an advantage over door-to-door surveys, as they prevent recall errors, and clinical details as well as laboratory results are readily available [4]. When easy access to care providers is guaranteed in a national health care system, a review of medical records can be utilized in understanding the epidemiology of certain disorders. To ensure efficiency and consistency of the overall study, the task of review- 
ing medical records was carried out by trained external reviewers. The goal of this study was to develop a protocol for and evaluate the validity and reliability of medical record survey for epilepsy by external reviewers, to support our future epidemiological study.

\section{MATERIALS AND METHODS}

\section{Subjects}

The two study hospitals, Korea University Hospital (K hospital located in Seoul) and Eulji University Hospital (E hospital located in a suburban area), are both tertiary centers. K hospital has an electronic medical record system, whereas $\mathrm{E}$ hospital does not.

Among those who were prescribed AEDs during the year 2009, 80 patients were randomly selected from the NHI claims data from both hospitals. To guarantee an adequate representation of various conditions, for each hospital, we sampled 18 patients coded as having epilepsy or seizure, 12 as having central nervous system (CNS) illness other than epilepsy or seizure, and 10 without any diagnostic codes related to CNS disease. AEDs included carbamazepine, clobazam, ethosuximide, gabapentin, lamotrigine, levetiracetam, oxcarbazepine, phenobarbital, phenytoin, pregabalin, topiramate, vigabatrin, valproate, and zonisamide. Clonazepam was excluded because it is rarely used as monotherapy for epilepsy and is more frequently used for non-epileptic purposes. Other anticonvulsants, including primidone, felbamate and tiagabin were not available in 2009 in Korea. The diagnostic codes indicating epilepsy or seizure included G40* (epilepsy), G41* (status epilepticus), F803 (Landau-Kleffner syndrome), and R56.8 (convulsion), based on the 10th version of the International Classification of Diseases (ICD10) and related health problems [5]. This study was approved by the Institutional Review Board of Korea University Hospital (AN10221-001).

\section{Procedures}

The investigators developed a case recording form (CRF) and common diagnostic algorithm, which was tested for consistency. The CRF consisted of two parts: the first part was a preliminary form for chart abstraction, written in layman terms and included guidelines for surveyors; the second part was the verification form, which included the diagnostic algorithm for epileptologists (Appendix).

Part one documented demographics, ICD-10 codes, department of primary physician, diagnosis, antiepileptic drugs prescribed, history of seizures, recurrence of seizures, age of onset, new-onset seizure or presence of any seizure during 2009, description of seizure type, cause of epilepsy, electroencephalog- raphy $(\mathrm{EEG})$ and brain imaging results. Age of onset was classified as $<12$ months, 12 months- 6 years, 6 years- 12 years, 12 years-18 years, 18 years- 30 years, 30 years- 60 years, and $>60$ years. EEG findings were categorized as normal, abnormal with focal epileptiform discharges, abnormal with generalized epileptiform discharges, and abnormal with non-epileptiform discharges.

Part two consisted of diagnosis, activity of epilepsy, cause, and classification of epilepsy. Diagnoses were categorized as: 1) epilepsy; 2) single seizure; 3) either epilepsy or seizure, unclear; 4) non-epileptic; and 5) either epileptic or non-epileptic, unclear. Epilepsy was defined as having two or more seizures during the patient's lifetime. In cases where it was uncertain whether he or she had single seizure or recurrent seizures, category 3 was assigned. Acute symptomatic seizures were categorized as 3 , even if they were recurrent. If the AED was being used for other identifiable reasons such as pain, the patients were classified as being category 4 . If the reason for prescribing an AED could not be determined, category 5 was assigned. Active epilepsy was defined as one or more seizures during 2009. The etiology was determined based on the clinical history, findings, brain imaging results, and EEG. If there were conflicting data, the etiology was determined by the clarity of the records and additional explanation from the treating physician. Non-specific imaging findings, such as small vessel disease, arachnoid cysts, venous anomalies, or diffuse atrophy were not considered as causes of epilepsy. Epilepsy was classified as: 1) generalized; 2) localization-related; 3) undetermined as to whether focal or generalized; 4) special syndrome, according to International League Against Epilepsy classification [6]; 5) and a lack of information for classification, based on the seizure type, syndrome diagnosis documented by the clinician, brain imaging, and EEG in the order of priority. Patients who had only generalized tonic-clonic seizures (GTCSs) with normal EEG and brain imaging were classified as 3. Patients who had only GTCSs with normal EEG but without any brain imaging results were classified as 5 .

For chart abstraction, we recruited certified health record administrators (HRAs) who were experienced in reviewing medical records for epidemiological studies and health registry. These were coders certified by the Department of Ministry and Health Care of Korea for abstracting and managing data from medical records. The HRAs were intensively trained to review medical records and extract data related to epilepsy for 8 hours. The didactic portion included an overall introduction, a general overview on epilepsy, medical terminology and abbreviations commonly used in physicians' notes, how to find test results, and other conditions in the differential diagnosis or that are treated with AEDs. The HRAs then received hands-on training in completing the CRFs while receiving feedback from the epileptologists, until they achieved a high level of concordance with the 
epileptologists. Training was provided at a third-party hospital. In this study, we assessed the validity and reliability of reviews performed by two HRAs (HRA-A and B), followed by verification by an external epileptologist (included SY Lee). HRA-A repeated the review at a 1-month interval to determine test-retest reliability. To establish a gold standard, each epileptologist performed both steps of chart abstraction and verification at his or her own hospital (E hospital, $\mathrm{K}$ hospital). In addition, alternative sources, such as the individual hospital data for patients with epilepsy or physician opinions were incorporated into the gold standard. The design of the study is summarized in Figure 1.

We analyzed the validity and reliability of the six items: diagnosis, activity of epilepsy, cause, classification (all verified by the epileptologist), new-onset epilepsy, and age of onset (obtained by the HRAs without verification by the epileptologist). The validity of the survey was estimated by measuring sensitivity and specificity. For multiple-choice items, we estimated the rate of the correct diagnosis. The level of reliability was estimated by kappa value, and graded to almost perfect $(\kappa=0.81-1.0)$, substantial ( $\kappa=0.61-0.80)$, moderate $(\kappa=0.41-0.60)$, fair $(\kappa=0.21$ 0.40 ), or slight ( $\kappa=0.0-0.2)$ agreement, according to the classifi-
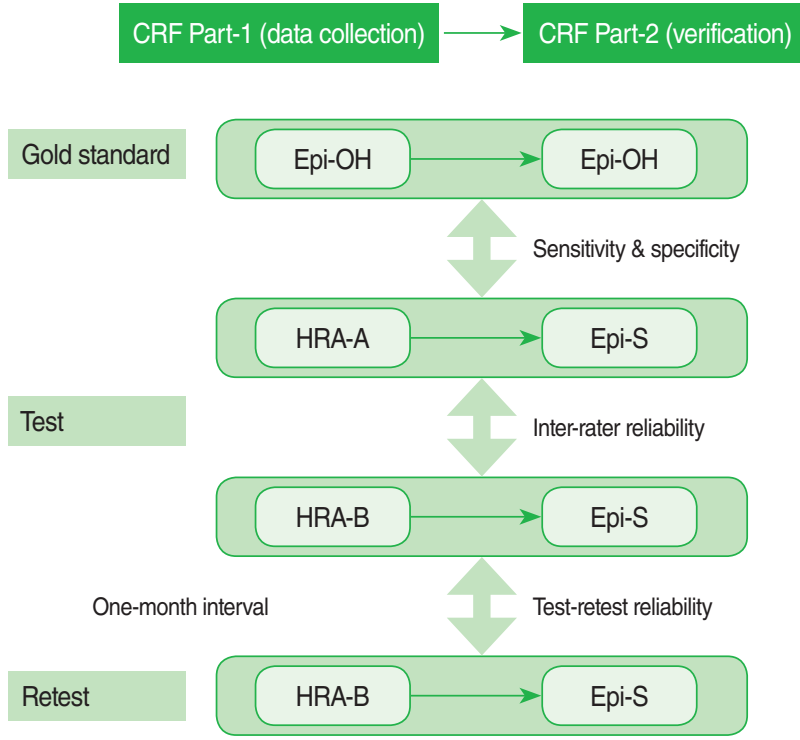

Figure 1. Study design for validation of medical record survey. The survey consisted of two-step data collection by certified health record administrators (HRAs) and then verification by the study epileptologist (Epi-S). For validation purposes, the gold standard was the conclusion each epileptologist made for his own hospital (Epi$\mathrm{OH})$, E or $\mathrm{K}$ hospital. In order to determine inter-rater reliability, two HRAs (HRA-A and HRA-B) reviewed medical records of the same patient while being blinded to each other. HRA-A repeated the reviews at a 1-month interval to determine test-retest reliability. CRF, case record form; HRA-A or B, health record administrator A or B; Epi-OH, epileptologist from the hospital; Epi-S, study epileptologist. cation system suggested by Landis and Koch [7]. Statistical analysis was performed using SPSS version 18.0 (SPSS Inc., Chicago, IL, USA).

\section{RESULTS}

\section{Baseline characteristics}

Of the total 80 subjects, 46 (57.5\%) were men and the mean age of subjects was 48.1 years old (range 9-88). Thirty-nine subjects had epilepsy, one had a single seizure, and two had seizure but it was unclear whether this recurred or not. Thirty-seven subjects received AEDs for non-epileptic causes; among them, 29 received AEDs for neuropathic pain, 4 for prophylaxis of seizure after brain insult or surgery, and the others for hemifacial spasm, facial nerve injury, oromandibular dyskinesia, or cramps. There was one case where the diagnosis was unclear (Table 1).

\section{Validity}

The sensitivity, specificity, and reliability of each surveyor are summarized in Table 2. For the diagnosis of epilepsy, the sensitivity was $97.6 \%$ for HRA-A and $100 \%$ for HRA-B, and specificity was $94.9 \%$ for HRA-A and $97.4 \%$ for HRA-B. For the activity of epilepsy, sensitivity was $100.0 \%$ for HRA-A and $97.0 \%$ for HRA-B, and specificity was $100.0 \%$ for both. For new-onset epilepsy, specificity was $90.9 \%$ for HRA-A and $100.0 \%$ for HRA-B, whereas sensitivity was $75.0 \%$ for HRA-A and $66.7 \%$ for HRA-B. The age of onset was correctly identified in $78.0 \%$ by HRA-A and $81.0 \%$ by HRA-B. The cause and classification of epilepsy were correctly identified and classified in $85.4 \%$

Table 1. Patient demographics $(n=80)$

\begin{tabular}{lr}
\hline Clinical characteristics of study patients & $n$ \\
\hline Age, mean (range) & $48.1(9-88)$ \\
Gender & 46 \\
Male & 34 \\
Female & \\
Diagnostic code & 36 \\
1. Epilepsy or seizure & 24 \\
2. CNS diseases other than epilepsy or seizure & 20 \\
3. Other than CNS diseases & 39 \\
Final diagnosis & 1 \\
1. Epilepsy & 2 \\
2. Single seizure & 37 \\
3. Either epilepsy or seizure, unclear & 4 \\
4. Non-epileptic & 29 \\
Prophylaxis for seizure & 4 \\
Pain & 1 \\
Other (hemifacial spasm, facial nerve injury, & \\
$\quad$ oromandibular dyskinesia, and cramps) & \\
5. Either epileptic or non-epileptic, unclear &
\end{tabular}

CNS, central nervous system. 
Table 2. Validity and reliability of the survey

\begin{tabular}{|c|c|c|c|c|c|c|c|c|}
\hline & \multicolumn{2}{|c|}{ Sensitivity (\%) } & \multicolumn{2}{|c|}{ Specificity (\%) } & \multicolumn{2}{|c|}{ Correct diagnosis (\%) } & \multicolumn{2}{|c|}{ Reliability: kappa [SE] } \\
\hline & A & B & A & $\mathrm{B}$ & A & B & Inter-rater (A-B) & Test-retest (A) \\
\hline Diagnosis & 97.6 & 100.0 & 94.9 & 97.4 & & & 0.907 [0.806-1.008] & $0.975[0.950-1.000]$ \\
\hline Activity & 100.0 & 97.0 & 100.0 & 100.0 & & & 0.932 [0.865-0.999] & $0.796[0.684-0.908]$ \\
\hline New onset & 75.0 & 66.7 & 90.9 & 100.0 & & & 0.728 [0.613-0.843] & $0.641[0.501-0.781]$ \\
\hline Age of onset & & & & & 78.0 & 81.0 & $0.753[0.674-0.832]$ & $0.697[0.616-0.778]$ \\
\hline Cause & & & & & 85.4 & 92.7 & $0.792[0.721-0.863]$ & $0.693[0.613-0.773]$ \\
\hline Classification & & & & & 90.2 & 92.9 & $0.864[0.771-0.957]$ & $0.787[0.670-0.904]$ \\
\hline
\end{tabular}

A, Health record administrator (HRA)-A; B, HRA-B; SE, standard error.

and $90.2 \%$ of cases, respectively for HRA-A, and $92.7 \%$ and $92.9 \%$, respectively for HRA-B.

Inter-rater reliability between HRA-A and B was almost perfect for the diagnosis $(\kappa=0.907)$, disease activity $(\kappa=0.932)$, and classification of epilepsy ( $\kappa=0.864)$. For new-onset epilepsy, age of onset, and cause of epilepsy, the reliability was substantial ( $\kappa=0.728, \kappa=0.753$, and $\kappa=0.792$, respectively).

The reliability of repeated examination was almost perfect for the diagnosis of epilepsy $(\kappa=0.975)$ and substantial for disease activity, new-onset epilepsy, age of onset, cause, and classification of epilepsy $(\kappa=0.796, \kappa=0.641, \kappa=0.697, \kappa=0.693$, and $\kappa=0.787$, respectively).

\section{DISCUSSION}

Our medical record review method demonstrated high levels of validity and consistency for the assessment of diagnosis, activity, and classification of epilepsy. The validity and reliability with regard to new-onset epilepsy, age of onset, and cause were acceptable.

The source of data from epidemiological studies may originate from direct population surveys, information or registries from physicians, medical records, or administrative data. Direct population surveys usually also involve interviews by non-physician surveyors or self-recorded questionnaires [8,9]. Some studies have validated questionnaires as a method to diagnose patients with epilepsy [8,9].

Reviewing medical records is highly useful to validate and supplement information from administrative data [8]. Reviewing of medical records is often performed by non-physician reviewers such as nurses or medical students, with or without a specialist's verification in epidemiological studies for epilepsy [9-11]. It would be ideal for the treating physicians to survey their own patients. However, not all of them are epileptologists and differences in experience would affect the consistency of the overall data. Although a standard protocol to confirm the diagnosis of epilepsy via medical records for epidemiological studies is not available, we are unaware of any other studies that have validated their tools. We showed that our non-physician reviewers could collect data that were sufficient for the epileptologists to diagnose epilepsy.

It is one of the limitations of our study that the gold standard was derived from medical records, lacking a face-to-face interview. We could not conclude which factors affected the validity, such as the type of medical record system or institution, specialties of physicians prescribing AEDs, or diagnostic codes, due to the small sample size.

Our results suggest that trained health care workers' review of medical records followed by verification by an epileptologist is a valid and consistent way to identify and characterize epilepsy patients. This allows large-scale epidemiological studies involving multiple distant locations to be performed at relatively low costs.

\section{ACKNOWLEDGEMENTS}

This study was supported by the Korean Epilepsy Society.

\section{CONFLICT OF INTEREST}

The authors have no conflicts of interest to declare for this study.

\section{REFERENCES}

1. Lee SY, Jung KY, Lee IK, Yi SD, Cho YW, Kim DW, et al. Prevalence of treated epilepsy in Korea based on national health insurance data. J Korean Med Sci 2012;27:285-290.

2. Wilchesky M, Tamblyn RM, Huang A. Validation of diagnostic codes within medical services claims. J Clin Epidemiol 2004;57:131-141.

3. Park SK, Cheong HK, Ki M, Son YM, Kim H. Assessment of the availability of health insurance data for epidemiologic study of childhood aseptic meningitis. Korean J Prev Med 2003;36:349-358. (Korean)

4. Thurman DJ, Beghi E, Begley CE, Berg AT, Buchhalter JR, Ding D, et al. Standards for epidemiologic studies and surveillance of epilep- 
sy. Epilepsia 2011;52 Suppl 7:2-26.

5. World Health Organization. ICD-10: international statistical classification of diseases and related health problems. Geneva: World Health Organization; 2005.

6. Proposal for revised classification of epilepsies and epileptic syndromes. Commission on Classification and Terminology of the International League Against Epilepsy. Epilepsia 1989;30:389-399.

7. Landis JR, Koch GG. The measurement of observer agreement for categorical data. Biometrics 1977;33:159-174.

8. Placencia M, Sander JW, Shorvon SD, Ellison RH, Cascante SM. Validation of a screening questionnaire for the detection of epileptic seizures in epidemiological studies. Brain 1992;115:783-794.

9. Ottman R, Barker-Cummings C, Leibson CL, Vasoli VM, Hauser WA, Buchhalter JR. Validation of a brief screening instrument for the ascertainment of epilepsy. Epilepsia 2010;51:191-197.

10. MacDonald BK, Cockerell OC, Sander JW, Shorvon SD. The incidence and lifetime prevalence of neurological disorders in a prospective community-based study in the UK. Brain 2000;123:665-676.

11. Christensen J, Vestergaard M, Olsen J, Sidenius P. Validation of epilepsy diagnoses in the Danish National Hospital Register. Epilepsy Res 2007;75:162-170. 


\section{Appendix}

\section{Epilepsy Case Recording Form (CRF)}

\begin{tabular}{|cc|c|c|c|}
\hline $\begin{array}{l}\text { Date } \\
\text { Survey }\end{array}$ & of & yr m d & $\begin{array}{c}\text { Institution } \\
\text { code }\end{array}$ & \\
\hline DOB & Gender & $\begin{array}{c}\text { Home address } \\
(\mathrm{City} / \mathrm{Gu})\end{array}$ \\
\hline $\mathrm{yr} \mathrm{m} \mathrm{d}$ & $\circ \mathrm{m} \circ \mathrm{f}$ & \\
\hline
\end{tabular}

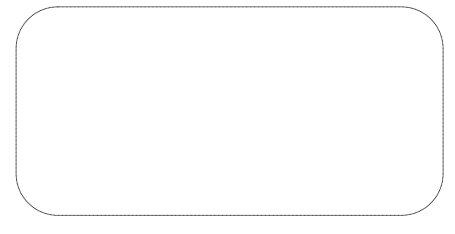

- Medical insurance claims data

\begin{tabular}{|c|c|c|c|c|c|c|c|c|c|}
\hline \multicolumn{2}{|c|}{ Diagnostic codes } & \multicolumn{4}{|c|}{ Primary diagnostic code } & \multicolumn{4}{|c|}{ Secondary diagnostic code } \\
\hline$\circ$ & Epilepsy or seizure & ○ & G40* & 0 & G41* & ० & $\mathrm{G} 40^{*}$ & ० & G41* \\
\hline$\circ$ & Other & $\circ$ & F803* & $\circ$ & R56* & $\circ$ & F803* & $\circ$ & $\mathrm{R} 56^{*}$ \\
\hline
\end{tabular}

If there was no codes for epilepsy or seizure, please document the codes given.

Antiepileptic medication can be multiple
- Carbamazepine
$\circ$ Lamotrigine
- Pregabalin
$\circ$ Phenytoin
- Clobazam
- Levetiracetam
- Topiramate
- Zonisamide
- Ethosuximide
- Oxcarbazepine
$\circ$ Vigabatrin
- Gabapentin
- Phenobarbital
- Valproate

Dept of the physician who prescribed the antiepileptic medication(s)

$\circ$ Neurology $\circ$ Neurosurgery $\circ$ Pediatrics $\circ$ Psychiatry $\circ$ Other

- Medical Records (clinical impression)

1)

3)
2)

4)

* in chronological order

- Identification of seizure It can be described as seizure, sz, fit, GTCS, aura, or a specific symptom.

For example: clonus, head drop, loss of consciousness, altered mental status, abnormal behavior, falls, etc.
1. Any history of seizure
$\circ$ Yes $\circ$ No $\circ$ Unclear

2. History of repetitive seizures

$\circ$ Yes $\circ$ No $\circ$ Unclear

3. What was the indication for prescribing antiepileptic medication if the patient did not have a seizure?

- Pain: abnormal sensation, paresthesias; headache, herpes zoster, diabetes, polyneuropathy, trigeminal neuralgia, etc.

- Hemifacial spasm

$\circ$ Obesity

Seizure prophylaxis - neurosurgery/head trauma /brain tumor

O Other

- Unclear
Possible seizure

antiepileptic medications were used despite lack of objective evidence as follows:

$\circ$ Loss of consciousness, LOC

Unusual behavior- amnesia, wandering, agitation

$\circ$ Falls

○ Tremor, twitching

$\circ$ Other 
- Details of Epilepsy

4. Did the patient have any seizure in 2009 ?

(if there was no description about seizure, consider as no seizure; An० Yes $\circ$ No aura should be considered a seizure)

\section{Did the seizures start in 2009 ?}

$\circ$ Yes $\circ$ No $\circ$ Undetermined

6. When did the seizures start?

$$
\begin{array}{ll}
\circ & <12 \text { months (infant) } \\
\circ & 12 \text { months } \sim<6 \text { years (toddler, preschool) } \\
\circ & 6 \sim 12 \text { years (elementary school) } \\
\circ & 12 \sim<18 \text { years (middle or high school age ) }
\end{array}
$$

$$
\begin{array}{ll}
\circ & 18 \sim<30 \text { years } \\
\circ & 30 \sim<60 \text { years } \\
\circ & >60 \text { years } \\
\circ & \text { Undetermined }
\end{array}
$$

7. Does the patient have auras? $\circ$ Yes $\circ$ No $\circ$ Undetermined

- can be multiple

$\begin{array}{cl}\circ & \text { Epigastric rising sensation } \\ \circ & \text { Nausea, vomiting } \\ \circ & \text { Visual } \\ \circ & \text { Auditory } \\ \circ & \begin{array}{l}\text { Dizziness (should be differentiated from adverse effect of } \\ \text { drug or nonspecific symptoms) }\end{array}\end{array}$

$\begin{array}{ll}\circ & \text { Memory flashbacks } \\ \circ & \text { (Un-)familiarity, jamais-vu, deja-vu } \\ \circ & \text { Paresthesias } \\ \circ & \text { Unexplainable feelings, cephalic aura } \\ \circ & \text { Other }\end{array}$

8. Type of seizure? can be multiple

- Generalized seizure (GS)

- Generalized tonic clonic, grand mal or GTCS

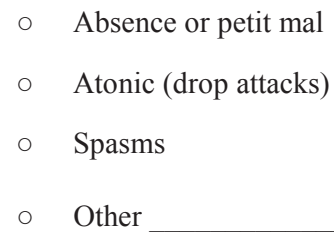

- Secondary generalized or secondary GTCS

- Partial seizure (PS), simple partial seizure (SPS), or complex partial seizure (CPS)

○ Myoclonus

○ Undetermined

9. Cause of epilepsy? based on past medical history and doctor's description; can be multiple

- Traumatic brain injury such as subdural hemorrhage (SDH), epidural hemorrhage (EDH), traumatic subarachnoid hemorrhage (tSAH), or contusion

- CNS infection

- Stroke, cerebral infarction, intracerebral hemorrhage (ICH), subarachnoid hemorrhage (SAH), Moyamoya disease

- Vascular malformation, arteriovenous malformation (AVM), cavernous angioma (CA)

- Brain tumor- oma, DNT, DNET

○ Degenerative disorders: dementia such as Alzheimer's (AD) or frontotemporal dementia (FTD)

- Perinatal injury or perinatal ischemia, cerebral palsy

- Congenital malformations of brain: malformation of cortical development (MCD), cortical dysplasia (CD), schizencephaly, pachygyria (lissencephaly), polymicrogyria, or heterotopia

○ Hippocampal sclerosis, HS, hippocampal atrophy

○ Other

- No identifiable cause (idiopathic or cryptogenic)

- Lack of information with regard to cause 
10. Epileptic syndrome? check all the clinical impressions in medical records; please document the final impression here given

$\circ$ Yes $\circ$ No

\section{I . Partial or focal epilepsy}

○ Localization-related epilepsy (LRE)

Occipital lobe epilepsy (OLE)

○ Temporal lobe epilepsy (TLE)

- $\quad$ Parietal lobe epilepsy (PLE)

○ Frontal lobe epilepsy (FLE)

$\circ \quad$ Benign rolandic epilepsy (BRE)

○ Childhood epilepsy with occipital paroxysms (CEOP)

- Panayiotopoulos syndrome

- Gastaut type

○ Other (anterior, posterior, left, right, central, insula etc)

$\circ$ Yes $\circ$ No

\section{Generalized epilepsy}

- Childhood absence epilepsy (CAE)

○ Juvenile absence epilepsy (JAE)

- Juvenile myoclonic epilepsy (JME)

- Idiopathic generalized epilepsy (IGE)

- Epilepsy with generalized tonic-clonic seizures (GTCS) on awakening

- Lennox-Gastaut syndrome (LGS)

- Infantile spasms or West syndrome

○ Epilepsy with myoclonic-astatic seizures (Doose syndrome)

○ Epilepsy with myoclonic absences (MAE)

- Early myoclonic encephalopathy (EME)

- Early infantile epileptic encephalopathy (EIEE) (Ohtahara syndrome)

- Benign neonatal convulsions

○ Benign myoclonic epilepsy in infancy

$\circ$ Yes $\circ$ No

\section{Undetermined whether partial or generalized}

- Severe myoclonic epilepsy in infancy (SMEI) (Dravet syndrome)

- Generalized epilepsy with febrile seizures plus (GEFS plus)

- Landau-Kleffner syndrome (LKS)

- Continuous spikes and waves during slow sleep (CSWS)

\section{$\circ$ Yes $\circ$ No IV. Special syndrome}

- Febrile convulsion (febrile seizure, FC)

- Seizures occurring only when there is an acute metabolic or toxic event due to factors such as alcohol, drugs, eclampsia, or nonketotic hyperglycemia

$\circ$ Yes $\circ$ No V. No documentation of an epileptic syndrome 


\section{- Diagnostic tests}

Search the interpretation results in order communication system or electric medical record first, then in the clinician's note.

11. Has the patient had an EEG? $\circ$ Yes $\circ$ No

11-1. What were the EEG $\circ$ Normal $\circ$ Abnormal results?

$\circ$ Yes $\circ$ No
- If an EEG was reported abnormal even once choose 'abnormal'. spikes, sharp waves, epileptiform discharges, rhythmic, ictal or epilepsy (do not include arrhythmic slowing)

- Generalized or diffuse (spike distribution)

- Localized or focal (suggestive of seizure focus)

Other abnormality

\section{Has the patient had a Brain MRI or CT?}
$\circ$ Yes $\circ$ No
MRI (date $\mathrm{yr}$ m)
$\circ$ Yes $\circ$ No
CT (date
yr
m)

12-1. What were the brain imaging results?

○ Normal ○ Abnormal

- If the report is too long, please attach the copy.

- Document only the results obtained prior to seizure onset; for example, traumatic brain injury after having a seizure would not be applicable.

Can be multiple

- Localized abnormality in cerebral hemisphere, cortex, or lobe

Localized abnormality in deep graymatter-basal ganglia, thalamus; brainstem-midbrain, pons, medulla; white matter

- Diffuse abnormalities: diffuse atrophy, hydrocephalus

○ Nonspecific: small vessel disease (SVD), unspecified bright opacities (UBO)

- Trauma: subdural hematoma (SDH), epidural hematoma (EDH), traumatic SAH, contusion

- Encephalitis, CNS infection

- Stroke, cerebral infarction, cerebral hemorrhage, ICH, SAH, Moyamoya disease

- Arteriovenous malformation (AVM), cavernous angioma (CA)

- Brain tumor- oma, DNT, DNET

- Degenerative disease: dementia such as Alzheimer's (AD) or frontotemporal dementia (FTD)

- Perinatal injury or perinatal ischemia, cerebral palsy

Congenital malformations of brain: malformation of cortical development (MCD), cortical dysplasia (CD), schizencephaly, pachygyria (lissencephaly), polymicrogyria, heterotopia

- Hippocampal sclerosis or atrophy

- Tuberous sclerosis or tubers

- Cerebromalacia, encephalomalacia or localized atrophy of unknown pathology

$\circ$ Other 


\section{Epileptologist's diagnosis}

\begin{tabular}{|c|c|}
\hline Date of Survey & yr m d \\
\hline Institution code & \\
\hline
\end{tabular}

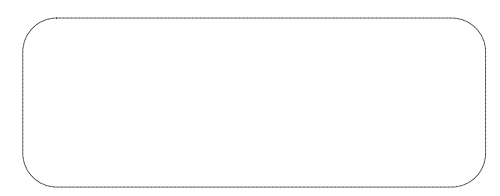

\section{- Diagnosis}

$\circ \quad$ Epilepsy

○ Single seizure

○ Seizure or Epilepsy, unclear

○ Other (nonseizure/nonepileptic)

$\circ \quad$ Epileptic or nonepileptic, unclear
Both 1\& 2: Yes

1: Yes, \& 2: No

1: Yes, \& 2: Undetermined

1: No or Undetermined, \& 3: Other cause

1 Undetermined, \& 3 Undetermined

\section{- Active epilepsy}

$\circ$ Yes $\circ$ No If yes in item 4, yes

- Cause of epilepsy (can be multiple)

Choose based on items 9 or 12. If there is conflicting data, 12 would hold priority, however, this would depend on the accuracy of the records and probability.

Cases marked as stroke, vascular malformation, tumor, congenital malformation on 9, but normal on 12 would be defined as undetermined

Cases marked as trauma on 9, but normal on 12 would be determined based on the accuracy of clinical information

- Cases marked as CNS infection or degenerative disease on 9, but normal on 12 will be determined as 9

- Cases marked as no specific cause on 9 and normal on 12 will be considered as idiopathic or cryptogenic

- Traumatic brain injury (mild head injuries are not to be considered a cause), SDH, EDH, traumatic SAH, contusion

- CNS infection

- Stroke, cerebral infarction, cerebral hemorrhage, $\mathrm{ICH}, \mathrm{SAH}$

- Arteriovenous malformation AVM, cavernous angioma CA

- Brain tumors

○ Degenerative disorders: dementia such as Alzheimer's (AD) or frontotemporal dementia (FTD)

- Perinatal injury or perinatal ischemia, cerebral palsy

- Congenital malformations of brain: malformation of cortical development (MCD), cortical dysplasia (CD), schizencephaly, pachygyria (lissencephaly), polymicrogyria, heterotopia

- Hippocampal sclerosis or atrophy

Other stroke), cortical thickening (cortical dysplasia or tumor))

(lesion of unknown pathology - cerebromalacia (trauma or

○ No identifiable cause (idiopathic or cryptogenic)

- Missing information on cause 


\section{- Classification of Epilepsy}

Classification is based on the seizure type (7-8), diagnosed syndrome (10) clinical information, brain imaging (12), and EEG (11) (in the order of priority)

-Seizure, or GTCS without any specific description -> undetermined

- 1) Localization-related epilepsy: the presence of one or more of the findings below without any findings to suggest generalized epilepsy

- Partial seizures

○ Aura

- Simple partial seizure, or complex partial seizure

- Secondary generalized or secondary GTCS

- Localization-related epilepsy (LRE), documented by physician

○ Temporal lobe epilepsy (TLE), documented by physician

○ $\quad$ Frontal lobe epilepsy (FLE), documented by physician

- Occipital lobe epilepsy (OLE), documented by physician

- $\quad$ Parietal lobe epilepsy (PLE), documented by physician

- Benign rolandic epilepsy (BRE), documented by physician

- Childhood epilepsy with occipital paroxysm (CEOP), documented by physician

○ Panayiotopoulos syndrome, documented by physician

- Gastaut type, documented by physician

- CT/MRI: localized abnormality in hemisphere $c f$. mild small vessel disease or unspecified bright opacity (UBO) is not considered as cause

- $\quad$ EEG: localized spikes

- Cause (history)

- Traumatic brain injury (trivial head trauma is not considered as cause) SDH, EDH, traumatic SAH, contusion

$\circ \quad$ CNS infection

- Stroke, cerebral infarction, cerebral hemorrhage, ICH, SAH

- Arteriovenous malformation AVM, cavernous angioma CA

- Brain tumor

- Degenerative disorders: dementia such as Alzheimer's (AD) or fontotemporal dementia (FTD)

- Perinatal injury or perinatal ischemia, cerebral palsy

- Congenital malformations of brain: malformation of cortical development (MCD), cortical dysplasia (CD), schizencephaly, pachygyria (lissencephaly), polymicrogyria, heterotopia

○ Hippocampal sclerosis or atrophy

- Tuberous sclerosis or tubers

- Other (lesion of unknown pathology - cerebromalacia (trauma or stroke), cortical thickening (cortical dysplasia or tumor)) 
2) Generalized epilepsy: the presence of one or more of the findings below and no findings to suggest - localization-related epilepsy (tuberous sclerosis or other focal cortical abnormalities does not rule out generalized epilepsy)

- Generalized seizure

$\begin{array}{cl}\circ & \text { Absence } \\ \circ & \text { Myoclonus } \\ \circ & \text { Atonic seizure } \\ \circ & \text { Spasm }\end{array}$

Childhood absence epilepsy (CAE), documented by physician

Juvenile absence epilepsy (JAE), documented by physician

Juvenile myoclonic epilepsy (JME), documented by physician

Idiopathic generalized epilepsy (IGE), documented by physician

Epilepsy with generalized tonic -clonic seizures (GTCS) on awakening, documented by physician

Lennox-Gastaut syndrome (LGS), documented by physician

Infantile spasms (IS) or West syndrome, documented by physician

Epilepsy with myoclonic-astatic seizures (Doose syndrome), documented by physician

Epilepsy with myoclonic absences (MAE), documented by physician

Early myoclonic encephalopathy (EME), documented by physician

Early infantile epileptic encephalopathy (EIEE) (Ohtahara syndrome), documented by physician

Benign neonatal convulsions, documented by physician

Benign myoclonic epilepsy in infancy, documented by physician

EEG: generalized spike

\section{3) Undetermined whether partial or generalized}

Severe myoclonic epilepsy in infancy (SMEI) (Dravet syndrome), documented by physician

Generalized epilepsy with febrile seizures plus (GEFS plus), documented by physician

Landau-Kleffner syndrome (LKS), documented by physician

Continuous spikes and waves during slow sleep (CSWS), documented by physician

Seizures without unequivocal generalized or focal features (including cases with only GTCS)

\section{4) Special syndrome}

Febrile convulsion (febrile seizure, FC)

Seizures occurring only when there is an acute metabolic or toxic event due to factors (such as alcohol, drugs, eclampsia, nonketotic hyperglycemia)

Other

5) Lack of information

Signature : 